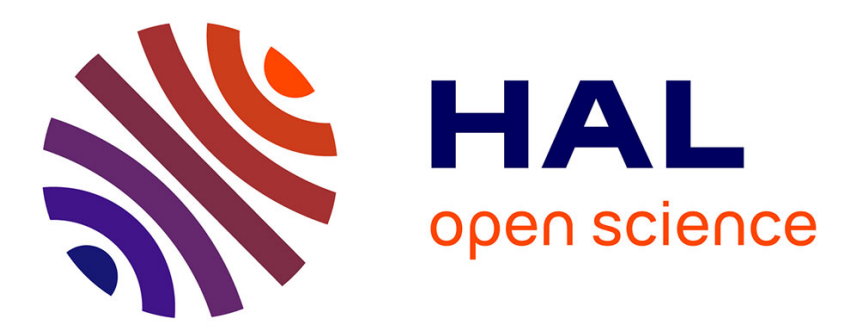

\title{
Humanized mouse models infected with human Plasmodium species for antimalarial drug discovery
}

Alicia Moreno-Sabater, Jean Louis Pérignon, Dominique Mazier, Catherine Lavazec, Valerie Soulard

\section{- To cite this version:}

Alicia Moreno-Sabater, Jean Louis Pérignon, Dominique Mazier, Catherine Lavazec, Valerie Soulard. Humanized mouse models infected with human Plasmodium species for antimalarial drug discovery. Expert Opinion on Drug Discovery, 2017, 13 (2), pp.131 - 140. 10.1080/17460441.2018.1410136 . hal-01827487

\section{HAL Id: hal-01827487 \\ https://hal.sorbonne-universite.fr/hal-01827487}

Submitted on 2 Jul 2018

HAL is a multi-disciplinary open access archive for the deposit and dissemination of scientific research documents, whether they are published or not. The documents may come from teaching and research institutions in France or abroad, or from public or private research centers.
L'archive ouverte pluridisciplinaire HAL, est destinée au dépôt et à la diffusion de documents scientifiques de niveau recherche, publiés ou non, émanant des établissements d'enseignement et de recherche français ou étrangers, des laboratoires publics ou privés. 


\title{
Humanized mouse models infected with human Plasmodium species for antimalarial drug discovery
}

\author{
Alicia Moreno-Sabater ${ }^{\mathrm{a}, \mathrm{b}}$, Jean Louis Pérignonc, Dominique Maziera, Catherine Lavazec ${ }^{\mathrm{d}, \mathrm{e}, \mathrm{f}}$ and Valerie Soulard ${ }^{\mathrm{a}}$ \\ aUPMC Faculte de Medecine - INSERM U1135, CNRS ERL 8255, Centre d'Immunologie et des Maladies Infectieuses (CIMI-Paris), Paris, Île-de-France \\ France; ${ }^{b}$ Assistance Publique - Hopitaux de Paris - Hôpitaux Universitaires Paris-Est - Site Saint-Antoine, Paris, île-de-France France; ${ }^{c}$ Assistance

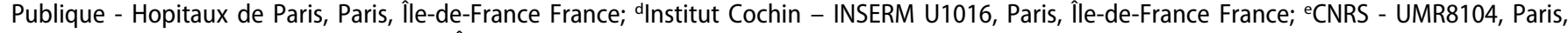 \\ France; fUniversite Paris Descartes, Paris, Île-de-France France
}

\begin{abstract}
Introduction: Efforts on malaria drug discovery are expected to increase in the coming years to achieve malaria eradication. Owing to the increasing number of new potential candidates together with the actual limitations of the primate models, humanized mouse models infected with human Plasmodium spp. $(\mathrm{HmHP})$ now appear as an alternative to the primate model.

Areas covered: The authors review the progress obtained in the $\mathrm{HmHP}$ in the last two decades, with a special emphasis of their input on the drug discovery pathway. The authors discuss the methodologies and strategies used in these models to obtain an accurate assessment of the compound activity and a reliable prediction of the human efficacious regimen.

Expert opinion: Research efforts have led us to an era in which $\mathrm{HmHP}$ can successfully be infected with $P$. falciparum, $P$ vivax and $P$. ovale. Furthermore, it is now a reality that the complete human cycle of $P$. falciparum can be obtained in $\mathrm{HmH}$. The $\mathrm{HmH} P$ has shown a real input mainly in the preclinical evaluation of new compounds against the erythrocytic stages of $P$. falciparum. However, further technical improvements are needed before $\mathrm{HmHP}$ may replace the primate model.
\end{abstract}

\section{KEYWORDS}

Antimalarial compounds; humanized mouse models; malaria eradication; preclinical pathway; $P$. falciparum; $P$. vivax; P. ovale

\section{Introduction}

There has been a dramatic increase in malaria drug discovery in the last 10 years and efforts are expected to increase in the coming years, for two main reasons. The first reason is that the present armamentarium against Plasmodium falciparum $(P$. falciparum), the parasite responsible for the large majority of malaria mortality (ca. 429,000 in $2015,92 \%$ in Africa, and $70 \%$ in children under 5 years old) [1], may soon become insufficient. Indeed, due to worldwide resistance to chloroquine, artemisinin-based combination therapies (ACTs) are the mainstay in the current malaria therapy. ACTs are recommended in most malaria-endemic countries as first-line treatment of uncomplicated $P$. falciparum malaria. However, it is well known that $P$. falciparum can become resistant against almost any drug that is in widespread use. Unfortunately, this has been confirmed 8 years ago, when first cases of reduced sensitivity of $P$. falciparum against artemisinins were reported from South-East Asia, which were characterized by a prolonged clearance time of the parasite [2]. In addition, laboratory strains have been rendered resistant to artemisinin, allowing the identification of a genetic marker of resistance and K13 propeller mutations were found to correlate with delayed in vivo clearance times [3]. The emergence and spread of resistance to antimalarial drugs are of major concern and are globally monitored, e.g. by the WorldWide Antimalarial Resistance Network, in order to inform the malaria community of potential efficacy loss of antimalarials [4]. This situation highlights the urgent need to find new drugs to treat the disease.

The second reason is that the objective of malaria drug discovery has become much more ambitious than circumventing the remarkable ability of malaria parasites to develop resistance to drugs. Indeed, 10 years ago (thus before the first report on reduced artemisinin efficacy), at the Malaria Forum in October 2007, the paradigm of malaria control and elimination has been extended to encompass an ultimate goal of malaria eradication [5]. The objective was thus not limited to $P$. falciparum, but was extended to all malaria parasites including Plasmodium vivax, which is responsible for $\sim 50 \%$ of the malaria cases outside sub-Sahara Africa. This position was rapidly supported by the World Health Organization, the Roll Back Malaria Partnership, and many other organizations and institutions. This call for malaria eradication led to a new research agenda laying out priorities with broader horizons [6]. Ideally, the objective is now Single Encounter Radical Cure and Prophylaxis (SERCaP), corresponding to a target product profile that includes (1) radical cure, defined as elimination of all parasites (including the long-lived hypnozoites of $P$. vivax or Plasmodium ovale), (2) suitability for mass administration (including administration to healthy subjects and the consequent need of a very good safety profile), and (3) prophylaxis for at least 1 month after treatment, to outlast the typical development period of Plasmodium parasites in Anopheline mosquitoes. 
Article highlights

- To face antimalarial drugs resistance and to achieve malaria eradication, continued efforts are mandatory to keep the antimalarial pipeline filled.

- For all the candidates that will emerge from the first screens, the major bottleneck will obviously be the translation into clinical development.

- In this context, limitations of the primate model become more important, leading to favor the $\mathrm{HmHP}$ in the preclinical pathway.

- Recent efforts carried out by the scientific community have permitted to proceed to an era in which different $\mathrm{HmHP}$ can successfully be infected with human $P$. falciparum, $P$ vivax and $P$. ovale, allowing the in vivo study of the parasite liver development for all of them and the complete human parasite development for $P$. falciparum.

- HmHPf-ES has shown to be an efficient tool to confirm in vivo antimalarial activity of new compounds, including against resistant parasites, and to help to predict human doses.

- Further technical improvements are needed before $\mathrm{HmHP}$ may replace the primate model in the drug discovery preclinical pathway.

This box summarizes key points contained in the article.

For these two reasons, the past decade has seen an unprecedented renewed focus on the discovery of new antimalarial drugs through extraordinary collaboration between academia (parasitologists, medicinal chemists, pharmacologists, clinicians) and industrial/private partnerships (e.g. Medicines for Malaria Venture). Cell-, chemistry-, and target-based approaches have already selected tens of thousands molecules $[7,8]$. The big challenge is to identify among them interesting compounds, advance them through preclinical and clinical development, to finally develop them into a registered antimalarial drug [9]. Indeed, several compounds recently entered Phase II testing or are in transition to Phase III evaluation $[7,10,11]$. Apart from two herbal medicines and the ozonide arterolane, preparations recently approved or in advanced stages of clinical development are mainly combinations of long known drugs. Given the high attrition rate even in advanced stages of development, only a few compounds with novel mechanisms will finally be introduced in therapy. In addition, none of the compounds corresponds to the ideal objective of SERCaP. Therefore, continued efforts are mandatory to keep the antimalarial pipeline filled, and for all the candidates that will emerge from the first screens, the major bottleneck will obviously be the translation into clinical development.

A major characteristic of research on drugs active against $P$. falciparum is that this parasite can be cultured, facilitating in vitro studies, whereas in vivo studies are restricted owing to strict host-parasite relationship. Indeed, promising antimalarial compounds are routinely assessed in the murine model infected with rodent Plasmodium spp. (most frequently Plasmodium berghei), with the obvious limitation of a different parasite and a different host. A next step may be provided by nonhuman primate models, which are much closer to malaria in the human host and are a valuable tool under some circumstances [12]. Splenectomized Aotus monkeys support growth of some $P$. falciparum strains and are mainly used to test novel vaccine candidates rather than drugs [9]. Till now, primate model was the only realistic possibility that could be proposed. However, the high costs and scarcity of primates have limited cohort sizes, which - in combination with the interindividual variability of an outbred species - limits the reproducibility of research findings. In addition, the use of primates, especially chimpanzees, has raised ethical concerns in many countries. The moratorium issued by the National Institutes of Health on 'nonessential' chimpanzee research is likely to further constrain aspects of malaria research in the future [13].

Humanized mouse models $(\mathrm{Hm})$ were initially developed to study viral infections with strict host specificity, most notably human immunodeficiency virus/acquired immunodeficiency syndrome [14]. Since then, the number of $\mathrm{Hm}$ for infectious diseases has rapidly expanded over the past years [15]. Applicated to human malaria, this approach led to graft immunodeficient mice with human erythrocytes or hepatocytes, thus allowing in vivo growth of $P$. falciparum and providing a promising tool for antimalarial drug discovery. The final goal was to obtain the full cycle of the human parasite in immunodeficient mice double grafted with both human red blood cells (HRBC) and human hepatocytes $(\mathrm{HH})$; ideally, adding the graft of human lymphoid cells would provide an attractive model for vaccine research.

The purpose of this review is to present improvements recently obtained with $\mathrm{Hm}$ infected with human Plasmodium species $(\mathrm{HmH} P)$ such as $P$. falciparum $(\mathrm{HmH} P f), P$. vivax $(\mathrm{HmH} P v)$, and $P$. ovale $(\mathrm{HmHPo})$, their input in the preclinical pathway of new compounds, their ability to confirm the in vivo activity of new compounds and to help to predict human doses. We discuss to what extent these models may contribute to the general endeavor to alleviate the threat of malaria parasites resistance against existing drugs and to design drugs fulfilling the objective of SERCaP. In our opinion, further technical improvements are needed before they may replace the primate model.

\section{HmHP for malaria research}

\subsection{The proof of concept}

Although great efforts have been employed to develop $\mathrm{Hm}$ for infectious diseases, the potential of $\mathrm{HmHP}$ for malaria research has been undervalued for many years (Figure 1). The initial development of HmHP was mainly sustained by severe combined immunodeficiency (SCID) mice, congenitally immunedeficient bg/nu/xid mice, or diabetes-susceptible nonobese diabetic (NOD)/SCID mice [16]. Using these precursor mouse models, several groups provided the proof of concept that $P$. falciparum liver stages (LS) could be obtained in immunodeficent mice grafted with $\mathrm{HH}$, to set up a $\mathrm{Hm}$ infected with $P$. falciparum LS (HmHPf-LS) $[17,18]$. In parallel, $P$. falciparum erythrocytic stages (ES) could be obtained in immunodeficient mice grafted with HRBC to obtain a $\mathrm{Hm}$ infected with $P$. falciparum ES (HmHPf-ES) [19,20].

Soon after, the HmHPf-ES, easier to obtain than the HmHPfLS, proved to be a useful tool to study immunological mechanisms and the activity of classical antimalarial drugs [21-23]. However, HmHPf-ES available at this time lacked reproducibility and showed a low rate of HRBC engraftment, 


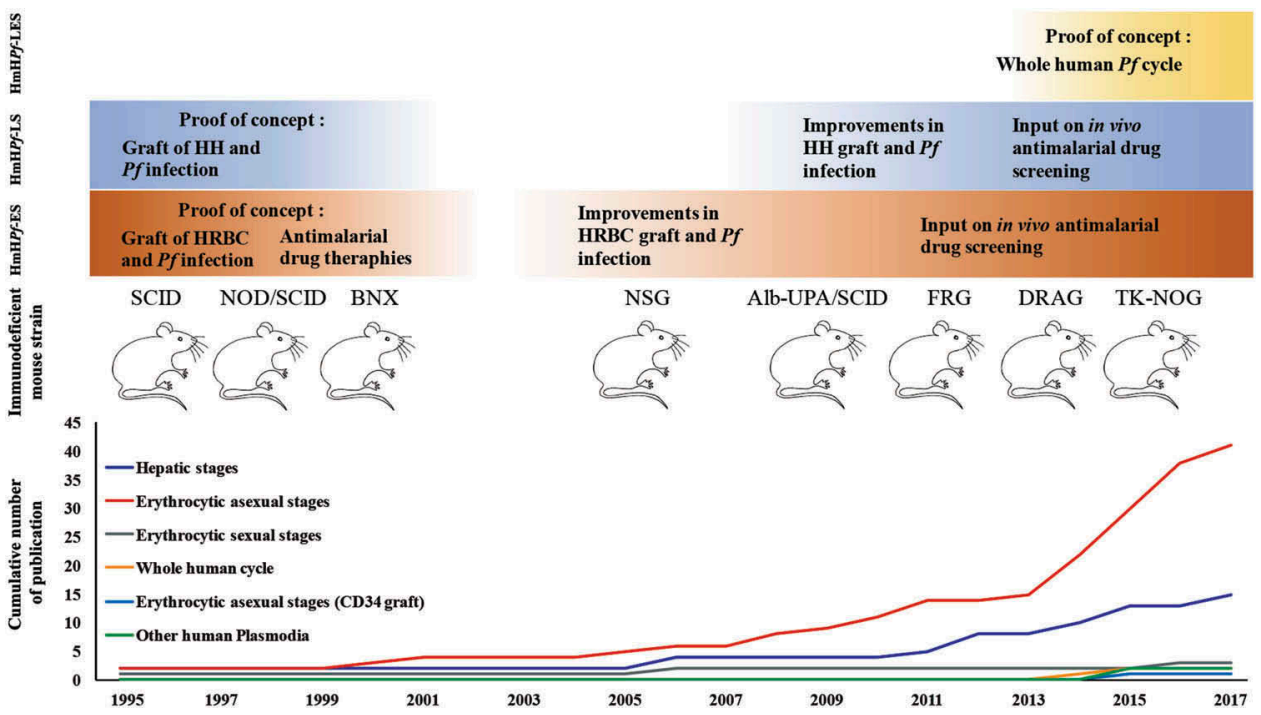

Figure 1. Evolution of humanized mouse models infected with human Plasmodium species (HmHP). The figure above shows the main improvements obtained during the last 20 years in the HmHP infected with P. falciparum (Pf) erythrocytic stages (HmHPf-ES), the liver stages (HmHPf-LS) or both stages (HmHPf-LES) comparing with the generation of new immunodeficient and transgenic mouse strains. The graph below shows the cumulative number of publications implicating the $\mathrm{HmHP}$ and it is correlated with the upper picture showing the progress in the improvements obtained during the last 20 years in the HmHP. Human hepatocytes (HH), Human red blood cells (HRBC).

leading to a disinterest of the scientific community. First reports pointed to the macrophagic system as responsible for HRBC clearance [24]. A significant improvement in the model reproducibility was obtained working with the NOD/ SCID mouse and the previously described immunomodulatory protocol [25]. Using this combination, all the P. falciparum ES, asexual and sexual parasites, were observed in the peripheral blood of mice infected with laboratory strains or with clinical isolates.

\subsection{Toward a HmHP for all parasite stages}

\subsubsection{HmHP for ES}

Although the HmHPf-LS fell into desuetude due to the technical difficulties and the scarce access to $\mathrm{HH}$, the interest in the HmHPf-ES was renewed thanks to the generation of new immunodeficient mouse strains (Figure 1). NOD/SCID ${ }^{\text {beta2m-/- }}$ mice [26] or NOD.Cg-Prkdc ${ }^{\text {scid }} / 12 \mathrm{rg}^{\text {tmiWjil }} / \mathrm{SzJ}$ (also named NSG mice), with reduced activity of natural killer cells (NK), allowed further improvement in the graft of HRBC and in the reproducibility of the HmHPf-ES [27-30]. Two different protocols should to be used to maintain $P$. falciparum infection: (1) a protocol requiring the use of immunochemical treatments to reduce HRBC clearance by macrophages or (2) a protocol requiring daily transfusions of $\mathrm{HRBC}$ and infection with an adapted strain of $P$. falciparum (i.e. a strain that has been selected after several successful infections in the HmHPf-ES).

One characteristic of the HmHPf-ES is that all the asexual stages are circulating in the peripheral blood, in contrast with the human infection where only the ring stages are circulating, whereas the mature stages cytoadhere to the vascular endothelium of organs. This suggests that parasite cytoadherence to the mouse vascular endothelium may not occur in the HmHPf-ES. Although some studies have observed a parasite accumulation in several organs as compared with the peripheral blood $[25,26]$, this may be related to a parasitized erythrocytes retention due to their lower deformability.

Using the NSG mouse strain, the complete sexual development of $P$. falciparum parasites has been obtained [31], confirming initial results obtained in the NOD/SCID strain [25]. Until then, the in vivo study of parasite sexual stages had been restricted to rodent Plasmodium spp., which show important biological differences with $P$. falciparum, or to splenectomised monkeys, precluding the use of these animals to address gametocytes' interactions with the spleen. One of the major challenges to obtain the sexual stages in the $\mathrm{HmHPf}$-ES was to reduce the rapid clearance of grafted HRBC (3-5 days) as gametocyte maturation needs at least 10 days. By modifying previously described immunosuppression protocols and using the NSG strain, it could be possible to obtain high gametocytemia and further characterize $P$. falciparum gametocytogenesis in this model. Interestingly, an enrichment of immature gametocytes was observed in the bone marrow and the spleen, suggesting a sequestration mechanism reminiscent to that observed in humans [31] and confirming that this is a valuable model to investigate the biology of the sexual forms in vivo.

While waiting a new generation of immunodeficient mouse strains with a reduced macrophage population, an alternative method to humanize mice with HRBC is the engraftment of human stem cells [32]. Recent progresses have established the proof of concept that the graft of stem cells from different sources allows the continuous production of HRBC in the peripheral blood of NSG mice. Generally, CD34+ cells mainly isolated from umbilical cord blood are injected intrahepatic or intravenously within immunodeficient mice. Recent promising improvements have shown that it is possible to generate HRBC permissive to different $P$. falciparum strains in NSG mice by transfecting the human stem cells with a plasmid that expresses IL-3 and erythropoietin [33]. Unfortunately, 
HRBC obtained by this procedure were only in low amounts (1.5-2.8\%), with a low parasite density. Consequently, new efforts should be concentrated on overcoming the short lifespan of those cells.

Thus, the increased numbers of studies using the HmHPf-ES in the last two decades witness its usefulness and its place in malaria research. This model has proved its value in immunological studies such as evaluating the potential inhibition of antibodies directed against proteins implicated in erythrocyte invasion by the merozoite cysteine-rich protective antigen [34], but its major contribution has been in the evaluation of new antimalarial drugs, which will be reviewed in the following section.

\subsection{2. $\mathrm{HmHP}$ for $\mathrm{LS}$}

The HmHPf-LS, long time forgotten, has also benefited from the generation of broader immunodeficient mice [35] (Figure 1). Efficient liver humanization relies simultaneously on the host immunodeficiency that facilitates xenotransplantation and on the selective elimination of endogenous murine hepatocytes in order to make room for the transplanted $\mathrm{HH}$ to repopulate the liver, prior to inoculation of human malaria sporozoites. Hence, $P$. falciparum LS development was first observed in homozygous Alb-UpA SCID mice [36,37] which express the hepatotoxic Urokinase Plasminogen Activator (UpA) transgene under the albumin (Alb) promoter leading to a constitutive loss of endogenous hepatocytes. AlbUpASCID mice are best engrafted at very young age (3-4 weeks) but given their low level of immunodeficiency, additional treatment to deplete NK cells and macrophages is required. Mice deficient for fumarylacetoacetate hydrolase $\left(\mathrm{FAH}^{-/-}\right)$with the broader immunodeficient $\mathrm{Rag}^{-/-}$, IL2R gamma null ${ }^{-1-}$ background (FRG) has been also used in malaria studies [38]. The $\mathrm{FAH}^{-1-}$ mice suffer from an acute liver failure that can be rescued by providing NTBC (2-(2nitro-4-trifluoromethylbenzoyl)-1, 3-cyclohexanedione) at regular intervals pre- and post-HH transplantation. The FRG mice backcrossed onto the NOD background (FRG NOD) allowed full maturation of the $P$. falciparum LS up to the generation of infectious hepatic merozoites [38]. Although this HmHPf-LS supported reproducible transition from LS to ES, the graft of HRBC was ephemeral and the ES were only observed when infected HRBC were cultured ex vivo. More recently, the TKNOG mouse strain, a model of inducible liver injury, has also proved to be valuable as a HmHPf-LS [39]. TK-NOG mice harbor the herpes simplex virus type 1 thymidine kinase (HSVtk) transgene under the albumin promoter onto the NSG background and upon a brief exposure to a nontoxic dose of gancyclovir, endogenous hepatocytes are depleted [40]. Numerous studies have then provided the proof of concept that HmHPf-LS are robust new experimental models to evaluate passive transfer therapies $[41,42]$, to study the identification of genetic determinants of parasite traits and adaptations [43], and to evaluate prophylaxis therapies [44]. Furthermore, LS development of other human Plasmodium species has been obtained with the FRG and TK-NOG mouse strains grafted with $\mathrm{HH}$ and infected with $P$. vivax and $P$. ovale (HmHPV-LS and HmHPo-LS), including the generation, persistence, and activation of hypnozoites [39,45].

\subsubsection{HmHP for LS and ES}

To obtain a $\mathrm{Hm}$ which harbors both the LS and ES of P. falciparum (HmHPf-LES), the scientific community has had to wait until the generation of new immunodeficient mice accept the simultaneous graft of $\mathrm{HH}$ and HRBCs. The proof of concept was showed up with the arrival of DR4.RagKO.IL2RY cKO.NOD (DRAG) mouse strain. To obtain mouse reconstitution with human liver and erythrocytes, DRAG mice were infused with HLA-matched human hematopoietic stem cells [46]. Although LS and ES could be obtained in this double $\mathrm{Hm}$, the level of humanization and the parasite load were very low, limiting the use of this model to malaria research.

Finally, the robust proof of concept that the whole human $P$. falciparum life cycle can be obtained in a double grafted model has been obtained in the TK-NOG mouse strain [39]. HRBC graft has been maintained for 5 weeks in these Pf-LSm through daily injections. In this double grafted mouse model, following inoculation of $P$. falciparum sporozoites, ES parasites could be detected in the blood from day 8 postinfection (parasitemia reached up to $1.52 \%$ ) and sexual stages after 21 days.

The achievement of the complete life cycle of $P$. ovale and $P$. vivax is the following step that could be obtained in a near future with progress in stem cells graft and in vivo production of human reticulocytes. So far, transition from $P$. vivax $L S$ to ES has been described in the FRG mice following transient enrichment of the infected liver-humanized mice with reticulocyteenriched HRBC, but no maintenance of a parasitemia has been reported [45].

\section{HmHP for antimalarial drug discovery}

The huge investment in new drug discovery to face artemisinin resistance and to achieve malaria eradication has also transformed the classical preclinical pathway. Drug efficacy, pharmacology, and toxicity are important parameters in the screening of compounds to proceed toward the clinical phase. In order to determine these parameters, new compounds are subjected to a series of in vitro and in vivo models with a decisive stage in the primate model, which is the most representative experimental model of the human infection, to confirm the therapeutic potential of compounds. Owing to the increased number of new potential candidates together with the actual limitations of the primate models, the progress realized in the $\mathrm{HmHP}$ makes them now appear as an alternative to the primate model.

\subsection{In vivo test used for antimalarial screening}

As seen in the previous section, different $\mathrm{HmHP}$ are now available but mainly the HmHPf-ES have been largely used in the preclinical evaluation of different antimalarial drugs targeting ES (Table 1). The efficacy of new compounds in the HmHPf-ES has usually been analyzed by transposing the classical tests set up to evaluate in vivo activity in rodent models, such as the 4 days Peter's test [72]. However, this test evaluates the suppressive effect of a compound, since the treatment is administered just after mouse infection with a rodent 
Table 1. Input of HmHPf-ES in antimalarial drug discovery.

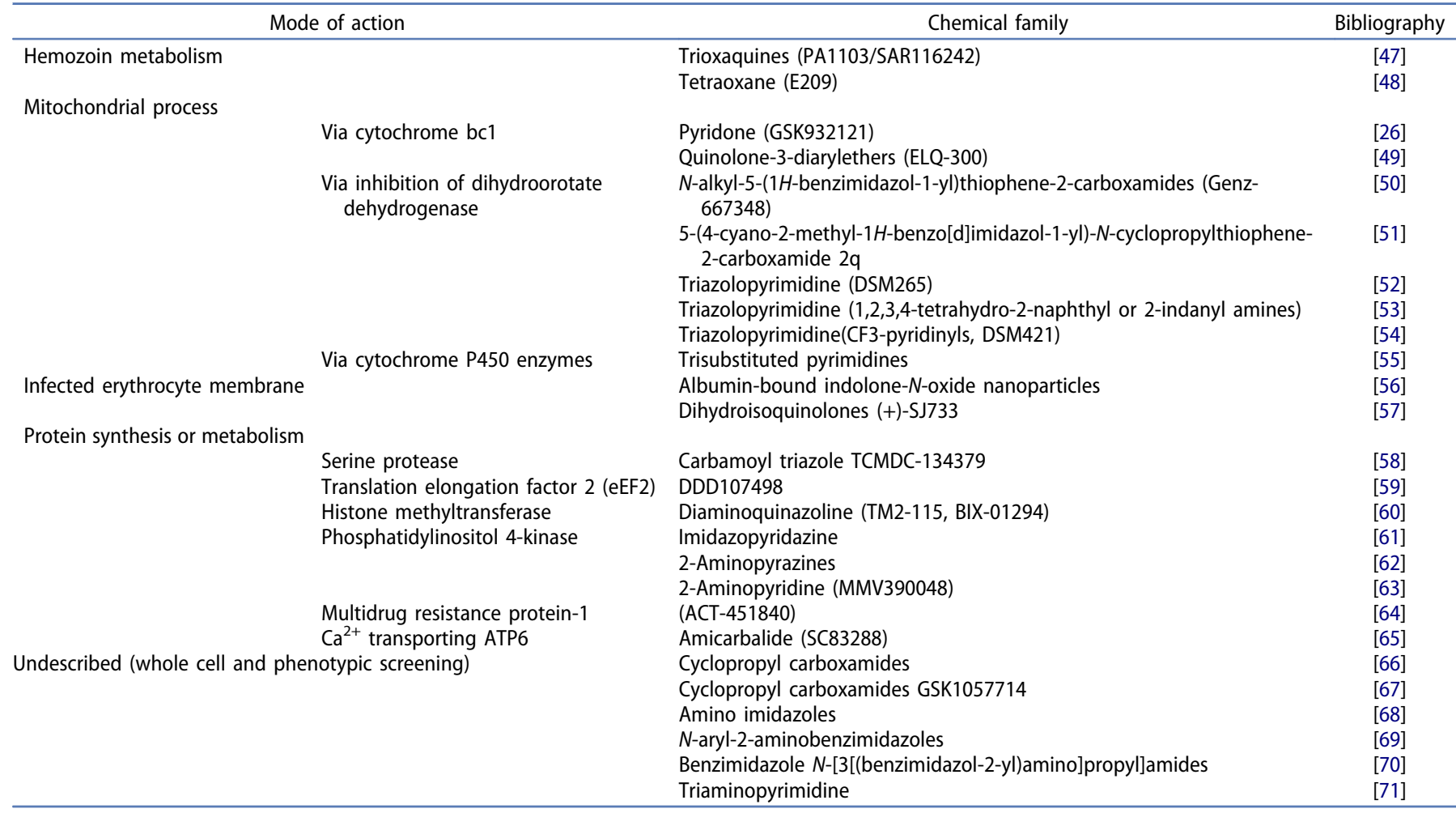

Possible mechanisms of action and the chemical family of compounds for which the in vivo activity against $P$. falciparum have been confirmed using the HmHPf-ES.

Plasmodium. The need of determining the therapeutic value of new compounds in the HmHPf-ES has led to introduce slight modifications in this test, such as administration of the therapeutic regimen once the parasitemia has developed [26,47]. In the actual context of antimalarial drug discovery and to support the global malaria eradication effort, there is a need for new medicines that can be given as a single dose to allow direct monitoring of administration and to ensure compliance. To this aim, the Thompson's test, which monitors the progress of infection following single-dose administration [73], has been used in the HmHPf-ES to assess the potential of radical cure of a compound after a single dose [49]. Most of the studies have also realized a 'dose-ranging test' to determine the classical parameters such as ED50 and ED 90 (50\% or $90 \%$ reduction in parasitemia) of these new compounds. Other parameters which are now being evaluated in the HmHPf-ES are the maximum kill rate after the first dose of treatment and the complete parasite clearance which allows to estimate the potential of parasite resistance development to a compound. Finally, some authors prefer to compare the parasite clearance rate induced by a compound with that induced by a classical antimalarial treatment $[26,30,47,63]$.

An added value of the HmHPf-ES is that it can also give precious information about compound activity against resistant strains. Thus, new compounds such as PA1103/ SAR116242, and SC81458 and SC83288 have shown an excellent activity in mice infected with the $P$. falciparum 3D7 strain as well as against the chloroquine-resistant $P$. falciparum W2 strain $[47,65]$. Other authors have taken advantage of the characteristic of this model - in which the different stages of the parasite are free in the circulation - to determine against which stage was acting the compound DDD107498, i.e. at the early ring stage or at the later mature schizont stage [59].

\subsection{Antimalarial activity of new promising compounds in the HmHPf-ES}

Using these tests, the HmHPf-ES has participated in the assessment of new compounds, most of them obtained from phenotypic or whole cell screenings; for some of them, the mechanism of action is known (Table 1). As the number of studies is consequent $[26,30,47-71,74]$, in this review, we focus on the most promising compounds. Among them, some compounds target the asexual ES with the aim to develop new therapies against simple or severe malaria access, such as PA1103/SAR116242, triaminopyrimidine, or SC81458 and SC83288 $[47,65,71]$. Other compounds also target the sexual ES such as (+)-SJ733, ACT-451840, E209 [48,57,64], or the LS which make them potential new therapeutic tools to provide radical cure and prophylaxis, such as the ELQ-300, DSM265, DDD107498, and MMV390048 [49,52,59,63]. It is interesting to highlight that in none of these works, the primate model was used to confirm the results obtained in the HmHPf-ES, which indirectly confirms the role of the latter model in the preclinical evaluation of a compound.

Compounds targeting different pathways such as protein synthesis, membrane transport, heme polymerization, or transcription have been evaluated in the HmHPf-ES to confirm the activity observed using the classical in vitro model and in vivo rodent Plasmodium spp. models (Table 1). When comparing efficacy parameters between different experimental models, efficacies observed for PA1103/SAR116242, triaminopyrimidine, 
ELQ-300, and DDD107498 compounds in rodent Plasmodium model and in HmHPf-ES were similar. In contrast, (+)-SJ733, ACT-451840, or E209 showed an efficacy higher in the HmHPfES than in the rodent Plasmodium model. It even happened that a compound was inactive in the rodent Plasmodium model infected with $P$. berghei whereas an excellent activity had been determined in vitro and could be confirmed using the HmHPfES, such as SC81458 and SC83288 [65]. Different therapeutic efficacy between Plasmodium species has been associated with charged compounds so that it could be hypothesized that discrepancies may be explained by selective uptake of charged compounds through parasite-induced channels in the host cell plasma membrane and species-specific characteristics of these new permeation pathways. These differences can be important in the progression of a compound throughout preclinical pathway toward the clinical phase. Since $P$. berghei is usually used in the first step of this screening, a reduced or a lack of activity in this model can stop the further development of a compound. Thus, HmHPf-ES may help to not unduly eliminate compounds during the preclinical pathway.

\subsection{Pharmacodynamic/Pharmacokinetic studies in the HmHPf-ES}

The HmHPf-ES has also opened new perspectives in pharmacokinetic (PK) studies together with pharmacodynamic (PD) studies that could allow a human dose prediction. In this context, the HmHPf-ES has participated in the preclinical assessment of dose-efficacy modeling, with the remarkable example of the ACT-451840 [64]. Briefly, assessment of PD/PK parameters in rodent Plasmodium infected and noninfected mice was used to model and predict dose regimens against the rodent Plasmodium. In parallel, PD/PK parameters were also evaluated in the HmHPf-ES. Finally, combining PD/PK obtained in the rodent and human Plasmodium models, a modeling approach was achieved to predict human efficacious exposure [64]. In an attempt to obtain a more accurate human dose prediction, other authors prefer to modify the 4day therapeutic assay by taking into account the PK parameters of the compound previously evaluated in mice. For example, because the half-life of DSM265 in mice is much shorter (2-4 h) than the one predicted in humans, this compound was dosed twice daily for 4 days in the HmHPf-ES [52]. Then, blood concentrations obtained using this therapeutic regimen in infected mice were used to determine the minimum concentration required for parasite killing.

\subsection{New HmHP for drug discovery}

The value of the HmHPf-ES has been also explored as a platform for testing drugs against $P$. falciparum erythrocytic sexual forms, those responsible for malaria transmission [31]. As a proof of concept, the effect of primaquine, which is currently the only licensed drug that has demonstrated efficacy to eliminate $P$. falciparum gametocytes in humans, was evaluated. After primaquine treatment, gametocytes were eliminated from peripheral blood and from sequestration sites, proving that the HmHPf-ES can be used for testing drugs against gametocytes. Primaquine can also eradicate the LS of
$P$. vivax and $P$. ovale. Its activity against different stages and different Plasmodium spp. makes primaquine an important antimalarial drug, but the risk of inducing hemolytic anemia in G6PD-deficient persons has reduced its utilization. Evaluation of drugs safer than primaquine in vivo is hampered by the fact that treatment with drugs known to cause hemolytic anemia in humans does not cause damage to mouse RBCs. To overcome this limitation, NSG mice have been humanized with G6PD-deficient HRBCs. In this new model, oral administration of primaquine induces a dose-dependent hemolytic response $[75,76]$ and treatment with drugs known to cause hemolytic toxicity (pamaquine, sitamaquine, tafenoquine, and dapsone) resulted in loss of G6PD-deficient HRBCs comparable to primaquine. In contrast, results obtained in this model show that $(-)-(R)$-primaquine may have a better safety margin than the racemate in human [77].

Finally, the HmHPf-LSm has recently proved its value to investigate liver parasites and facilitate the discovery of drugs targeting this stage [44]. For an easier evaluation of parasite infection in liver, mice are infected using a GFP-luc transgenic $P$. falciparum strain and drug efficacy can be monitored without killing mice or using complicated and timeconsuming histological methods. The accuracy of this model has been evaluated after treatment with different antimalarial drugs. Parasite clearance could be observed after atovaquone treatment or using a combination of $\mathrm{Bcl}-2$ inhibitors and P53 agonists treatments [44].

\section{Conclusions}

Recent efforts carried out by the scientific community have permitted to proceed to an era in which $\mathrm{HmHP}$ can successfully be infected with human Plasmodium spp.: $P$. falciparum, $P$ vivax, and $P$. ovale. Furthermore, it is now a reality that the complete human cycle of $P$. falciparum can be obtained in $\mathrm{HmH} P$, ultimately allowing to reproducibly obtain transmission to mosquitoes. The HmHPf-ES has shown a real input in the preclinical evaluation of new compounds against the ES: its efficacy is similar to that of the rodent Plasmodium model, it allows to study compound activity against $P$. falciparum resistant strains and to realize a prediction of the human dose by taking into account the PK parameters.

\section{Expert opinion}

The present review of $\mathrm{HmHP}$ suggests that, theoretically, these models may replace the rodent Plasmodium model in the preclinical pathway, thereby reducing the risk of unduly eliminate compounds inactive against rodent Plasmodium spp. However, considering 10 years of experience in the HmHPfES, it appears that compounds with a species-specific activity are more an exception than a rule. Furthermore, for economic and technical reasons, the use of the HmHPf-ES is reduced to a limited number of laboratories. Consequently, the rodent Plasmodium model keeps a main place in the initial step of the preclinical pathway. Nonetheless, $\mathrm{HmHP}$ is a valuable tool in case of discrepancies between in vitro data and those obtained in the rodent model. In addition, there is an important advantage of the HmHPf-ES, which has been largely 
underexploited: its ability to evaluate compound activity against strains artemisin resistant or against clinical isolates with in vivo reduced clearance. Thus, it is regrettable that most studies have evaluated compounds activity against a sensitive mouse-adapted $P$. falciparum strain and only a reduced number of works have explored the activity against chloroquineresistant strains.

In the present context where an unprecedented number of new compounds must be evaluated to confirm their in vivo activity against $P$. falciparum, the ethical, economical, and practical limitations of the primate model become more important, leading to favor the HmHPf-ES. The input of the primate model in the preclinical pathway is primary to confirm the efficacy of compounds and to predict the human dose, as primate metabolism is closer to human metabolism than rodent metabolism. Considerable efforts have been done to overcome these differences and PD/PK studies are now realized in the HmHPf-ES to obtain a more accurate prediction. Preliminary studies comparing the data obtained in the $\mathrm{HmHP}$ and in the human challenge model are encouraging [78]. Nonetheless, the proof of concept of this modelization has not yet been demonstrated, as none of the compounds has reached the clinical phase in human. Meanwhile, several technical improvements of the HmHPf-ES should contribute to the final objective of replacing the primate model.

A technical improvement concerns the tests used to evaluate the activity of compounds. Till now, the modified 4-day test [72] or the Thompson's test have been accommodated to the HmHPfES. However, these tests have been set up to evaluate the activity of compounds against the rodent Plasmodium infection. The biology between rodent and human Plasmodium spp. diverges in many aspects but especially in time that parasites need to achieve their erythrocytic cycle: $P$. falciparum requires $48 \mathrm{~h}$ whereas rodent Plasmodium spp. need only $24 \mathrm{~h}$. Considering that PK parameters are faster in mouse than in humans, there is an imbalance between parasite maturation and mouse PK. This imbalance might explain that for some antimalarials with a short half-life such as artesunate, dosages of $50 \mathrm{mg} / \mathrm{kg}$ are needed in the HmHPf-ES to clear parasitemia while the human dose chosen is $4 \mathrm{mg} / \mathrm{kg}$. Thus, the recently introduced test in which the compound is administrated twice a day could counteract rapid mouse PK. Consequently, new studies should tend to use this 'twice a day - 4 days test' or 'twice a day - Thompson's test.' Another technical improvement will be to carry out PD/PK studies in mouse grafted with $\mathrm{HH}$ to mimic $\mathrm{HH}$ metabolism.

Further improvements will require the generation of new immunodeficient mice. Protocols to obtain HRBC humanization are still a constraint. It is not known whether these protocols could alter the compound evaluation. Daily transfusion of HRBC may induce a dilution factor during parasitemia assessment and the use of immunosuppressive treatments could interact with drug activity or reduce the physiological clearance of parasites. As it is now clearly demonstrated that phagocyte population plays a major role in HRBC clearance, it is mandatory to generate new immunodeficient mice with a reduced macrophagic population. In parallel, efforts should be made to improve the graft of CD34 cells and the production of HRBC in the $\mathrm{HmHP}$. This methodology could allow the in vivo generation of reticulocytes and the setup of a $\mathrm{Hm}$ for $P$. vivax and $P$. ovale ES.
Correcting the lack of cytoadherence in the HmHPf-ES, as compared with human infection, will also require the generation of new immunodeficient mice. A first objective is to reduce the difference between the parasite biomass that a compound must face in the HmHPf-ES as compared to human infection. Indeed, the parasite biomass in the HmHPf-ES, where parasites freely circulate, may be quite less than that in human infection, where the parasites cytoadhere throughout the vascular system. Consequently, it is reasonable to wonder whether the dose determined in the HmHPf-ES could face the parasite biomass in humans. This question has further consequences when the ability of $P$. falciparum to develop resistances is taken into account. A second objective is to set up a new animal model for in vivo studies of mechanisms leading to the onset of severe malaria. The lack of cytoadherence is most probably due to the lack of interactions between $P$. falciparum antigens and mouse vascular adhesion receptors. Therefore, new immunodeficient mice transgenic for human vascular adhesion molecules could overcome this limitation.

After two decades of continuous developments and progress obtained in the $\mathrm{HmHP}$, the state of the art shows that it is now a valuable and accurate model for antimalarial drug discovery. The present review shows its input in the preclinical pathway of new compounds, its ability to confirm their in vivo activity including against resistant parasites and to help to predict human doses. In our opinion, further technical improvements are needed before $\mathrm{HmHP}$ may replace the primate model.

\section{Funding}

This manuscript has not been funded.

\section{Declaration of interest}

The authors have no relevant affiliations or financial involvement with any organization or entity with a financial interest in or financial conflict with the subject matter or materials discussed in the manuscript. This includes employment, consultancies, honoraria, stock ownership or options, expert testimony, grants or patents received or pending, or royalties. Peer reviewers on this manuscript have no relevant financial or other relationships to disclose

\section{References}

Papers of special note have been highlighted as either of interest $(\cdot)$ or of considerable interest (*) to readers.

1. WMR. World malaria report 2016. 2016. Available from: http:// www.who.int/malaria/publications/world-malaria-report-2016/ report/en/.

.- The World Malaria Report 2016 summarizes information received from malaria-endemic countries and other sources and updates the analyses presented in the $\mathbf{2 0 1 5}$ report.

2. Dondorp AM, Nosten F, Yi P, et al. Artemisinin resistance in Plasmodium falciparum malaria. N Engl J Med. 2009 Jul 30;361 (5):455-467. PubMed PMID: 19641202; PubMed Central PMCID: PMCPMC3495232.

- This manuscript shows initial cases of reduced $P$. falciparum susceptibility to artesunate in western Cambodia as compared with northwestern Thailand.

3. Ariey F, Witkowski B, Amaratunga C, et al. A molecular marker of artemisinin-resistant Plasmodium falciparum malaria. Nature. 2014 Jan 2;505(7481):50-55. PubMed PMID: 24352242; PubMed Central PMCID: PMCPMC5007947. 
- Strong correlations between the presence of a mutant allele, in vitro parasite survival rates and in vivo parasite clearance rates indicated that K13-propeller mutations are important determinants of artemisinin resistance.

4. Verlinden BK, Louw A, Birkholtz LM. Resisting resistance: is there a solution for malaria? Expert Opin Drug Discov. 2016;11(4):395-406. PubMed PMID: 26926843.

5. Tanner M, De Savigny D. Malaria eradication back on the table. Bull World Health Organ. 2008 Feb;86(2):82. PubMed PMID: 18297155; PubMed Central PMCID: PMCPMC2647379.

6. Alonso PL, Brown G, Arevalo-Herrera M, et al. A research agenda to underpin malaria eradication. PLoS Med. 2011 Jan 25;8(1): e1000406. PubMed PMID: 21311579; PubMed Central PMCID: PMCPMC3026687.

-. This report shows the need of a sustained commitment from local communities, civil society, policy leaders, and the scientific community, together with a massive effort to build a strong base of researchers from the endemic areas to malaria eradication.

7. Flannery EL, Chatterjee AK, Winzeler EA. Antimalarial drug discovery - approaches and progress towards new medicines. Nat Rev Microbiol. 2013 Dec;11(12):849-862. PubMed PMID: 24217412; PubMed Central PMCID: PMCPMC3941073.

.- This review shows the cell-, chemistry-, and target-based approaches used to discover new drug candidates that are currently in clinical trials or undergoing preclinical testing.

8. Flannery EL, Chatterjee AK, Winzeler EA. Antimalarial drug discovery - approaches and progress towards new medicines. Nat Rev Microbiol. 2017 Jul 24. DOI:10.1038/nrmicro.2017.88. PubMed PMID: 28736448.

9. Held J, Kreidenweiss A, Mordmuller B. Novel approaches in antimalarial drug discovery. Expert Opin Drug Discov. 2013 Nov;8 (11):1325-1337. PubMed PMID: 24090219.

10. Anthony MP, Burrows JN, Duparc S, et al. The global pipeline of new medicines for the control and elimination of malaria. Malar J. 2012 Sep 7;11:316. PubMed PMID: 22958514; PubMed Central PMCID: PMCPMC3472257.

- This manuscript reviews the global portfolio of new medicines in development against malaria.

11. Held J, Jeyaraj S, Kreidenweiss A. Antimalarial compounds in Phase II clinical development. Expert Opin Investig Drugs. 2015 Mar;24 (3):363-382. PubMed PMID: 25563531.

- This manuscript reviews the global portfolio of new medicines in development against malaria.

12. Beignon AS, Le Grand R, Chapon C. In vivo imaging in NHP models of malaria: challenges, progress and outlooks. Parasitol Int. 2014 Feb;63(1):206-215. PubMed PMID: 24042056.

13. Wadman M. Animal rights: chimpanzee research on trial. Nature. 2011 Jun 15;474(7351):268-271. PubMed PMID: 21677722.

14. Akkina R, Allam A, Balazs AB, et al. Improvements and limitations of humanized mouse models for HIV research: NIH/NIAID "meet the experts" 2015 workshop summary. AIDS Res Hum Retroviruses. 2016 Feb;32(2):109-119. PubMed PMID: 26670361; PubMed Central PMCID: PMCPMC4761823.

.. This review shows improvements and limitations of humanized mouse models and the mouse strains used in humanized mouse models.

15. Brehm MA, Wiles MV, Greiner DL, et al. Generation of improved humanized mouse models for human infectious diseases. J Immunol Methods. 2014 Aug;410:3-17. PubMed PMID: 24607601; PubMed Central PMCID: PMCPMC4155027.

16. Moreno A, Perignon JL, Morosan S, et al. Plasmodium falciparuminfected mice: more than a tour de force. Trends Parasitol. 2007 Jun;23(6):254-259. PubMed PMID: 17434343.

- This review illustrates the first humanized mouse models used in malaria studies.

17. Butcher GA, Couchman AF, Van Pelt JF, et al. The SCID mouse as a laboratory model for development of the exoerythrocytic stages of human and rodent malaria. Exp Parasitol. 1993 Sep;77(2):257-260. PubMed PMID: 8375494.
18. Sacci JB Jr., Schriefer ME, Resau JH, et al. Mouse model for exoerythrocytic stages of Plasmodium falciparum malaria parasite. Proc Natl Acad Sci U S A. 1992 May 1;89(9):3701-3705. PubMed PMID: 1570289; PubMed Central PMCID: PMCPMC525558.

19. Moore JM, Kumar N, Shultz LD, et al. Maintenance of the human malarial parasite, Plasmodium falciparum, in scid mice and transmission of gametocytes to mosquitoes. J Exp Med. 1995 Jun 1;181 (6):2265-2270. PubMed PMID: 7760012; PubMed Central PMCID: PMCPMC2192049.

20. Tsuji M, Ishihara $C$, Arai $S$, et al. Establishment of a SCID mouse model having circulating human red blood cells and a possible growth of Plasmodium falciparum in the mouse. Vaccine. 1995;13 (15):1389-1392. PubMed PMID: 8578814.

21. Badell E, Oeuvray C, Moreno A, et al. Human malaria in immunocompromised mice: an in vivo model to study defense mechanisms against Plasmodium falciparum. J Exp Med. 2000 Dec 4;192 (11):1653-1660. PubMed PMID: 11104807; PubMed Central PMCID: PMCPMC2193098.

22. Druilhe $P$, Spertini $F$, Soesoe $D$, et al. A malaria vaccine that elicits in humans antibodies able to kill Plasmodium falciparum. PLoS Med. 2005 Nov;2(11):e344. PubMed PMID: 16262450; PubMed Central PMCID: PMCPMC1277929.

23. Moreno A, Badell $\mathrm{E}$, Van Rooijen $\mathrm{N}$, et al. Human malaria in immunocompromised mice: new in vivo model for chemotherapy studies. Antimicrob Agents Chemother. 2001 Jun;45(6):1847-1853. PubMed PMID: 11353636; PubMed Central PMCID: PMCPMC90556.

24. Moreno Sabater A, Moreno M, Moreno FJ, et al. Experimental infection of immunomodulated NOD/LtSz-SCID mice as a new model for Plasmodium falciparum erythrocytic stages. Parasitol Res. 2005 Jan;95(2):97-105. PubMed PMID: 15592938.

25. Moreno A, Ferrer E, Arahuetes $S$, et al. The course of infections and pathology in immunomodulated NOD/LtSz-SCID mice inoculated with Plasmodium falciparum laboratory lines and clinical isolates. Int J Parasitol. 2006 Mar;36(3):361-369. PubMed PMID: 16443227.

26. Angulo-Barturen I, Jimenez-Diaz MB, Mulet T, et al. A murine model of falciparum-malaria by in vivo selection of competent strains in non-myelodepleted mice engrafted with human erythrocytes. PLoS One. 2008 May 21;3(5):e2252. PubMed PMID: 18493601; PubMed Central PMCID: PMCPMC2375113.

27. Arnold L, Tyagi RK, Meija $P$, et al. Further improvements of the $P$. falciparum humanized mouse model. PLoS One. 2011 Mar 31;6(3): e18045. PubMed PMID: 21483851; PubMed Central PMCID: PMCPMC3069031.

28. Arnold L, Tyagi RK, Mejia P, et al. Analysis of innate defences against Plasmodium falciparum in immunodeficient mice. Malar J. 2010 Jul 9;9:197. PubMed PMID: 20618960; PubMed Central PMCID: PMCPMC2914061.

29. Chen Q, Amaladoss A, Ye W, et al. Human natural killer cells control Plasmodium falciparum infection by eliminating infected red blood cells. Proc Natl Acad Sci U S A. 2014 Jan 28;111(4):1479-1484. PubMed PMID: 24474774; PubMed Central PMCID: PMCPMC3910619.

- This report shows the role of natural killer cells in mouse humanization with human red blood cells.

30. Jimenez-Diaz MB, Mulet T, Viera S, et al. Improved murine model of malaria using Plasmodium falciparum competent strains and nonmyelodepleted NOD-acid IL2Rgammanull mice engrafted with human erythrocytes. Antimicrob Agents Chemother. 2009 Oct;53 (10):4533-4536. PubMed PMID: 19596869; PubMed Central PMCID: PMCPMC2764183.

31. Duffier $Y$, Lorthiois $A$, Cistero $P$, et al. A humanized mouse model for sequestration of Plasmodium falciparum sexual stages and in vivo evaluation of gametocytidal drugs. Sci Rep. 2016 Oct 12;6:35025. PubMed PMID: 27731362; PubMed Central PMCID: PMCPMC5059736.

32. Noulin F. Malaria modeling: in vitro stem cells vs in vivo models. World J Stem Cells. 2016 Mar 26;8(3):88-100. PubMed PMID: 27022439; PubMed Central PMCID: PMCPMC4807312.

- This manuscript reviews the different protocols available to study human Plasmodium species either by using stem cell or alternative animal models. 
33. Amaladoss A, Chen $Q$, Liu $M$, et al. De novo generated human red blood cells in humanized mice support Plasmodium falciparum infection. PLoS One. 2015;10(6):e0129825. PubMed PMID: 26098918; PubMed Central PMCID: PMCPMC4476714.

34. Dreyer AM, Matile $H$, Papastogiannidis $P$, et al. Passive immunoprotection of Plasmodium falciparum-infected mice designates the CyRPA as candidate malaria vaccine antigen. J Immunol. 2012 Jun 15;188(12):6225-6237. PubMed PMID: 22593616.

35. Good MF, Hawkes MT, Yanow SK. Humanized mouse models to study cell-mediated immune responses to liver-stage malaria vaccines. Trends Parasitol. 2015 Nov;31(11):583-594. PubMed PMID: 26458783.

- This review is focused on the complexities of T-cell development in a mouse humanized with both a lymphoid system and hepatocytes.

36. Morosan S, Hez-Deroubaix S, Lunel F, et al. Liver-stage development of Plasmodium falciparum, in a humanized mouse model. J Infect Dis. 2006 Apr 1;193(7):996-1004. PubMed PMID: 16518762.

37. Sacci JB Jr., Alam U, Douglas D, et al. Plasmodium falciparum infection and exoerythrocytic development in mice with chimeric human livers. Int J Parasitol. 2006 Mar;36(3):353-360. PubMed PMID: 16442544.

38. Vaughan AM, Mikolajczak SA, Wilson EM, et al. Complete Plasmodium falciparum liver-stage development in liver-chimeric mice. J Clin Invest. 2012 Oct;122(10):3618-3628. PubMed PMID: 22996664; PubMed Central PMCID: PMCPMC3461911.

39. Soulard V, Bosson-Vanga $H$, Lorthiois $A$, et al. Plasmodium falciparum full life cycle and Plasmodium ovale liver stages in humanized mice. Nat Commun. 2015 Jul 24;6:7690. PubMed PMID: 26205537; PubMed Central PMCID: PMCPMC4525212.

- This manuscript shows the proof of concept that the whole $P$. falciparum human cycle can be obtained in a humanized mouse model.

40. Hasegawa M, Kawai K, Mitsui T, et al. The reconstituted 'humanized liver' in TK-NOG mice is mature and functional. Biochem Biophys Res Commun. 2011 Feb 18;405(3):405-410. PubMed PMID: 21238430; PubMed Central PMCID: PMCPMC3648850.

41. Foquet L, Hermsen CC, Van Gemert GJ, et al. Vaccine-induced monoclonal antibodies targeting circumsporozoite protein prevent Plasmodium falciparum infection. J Clin Invest. 2014 Jan;124 (1):140-144. PubMed PMID: 24292709; PubMed Central PMCID: PMCPMC3871238.

42. Sack BK, Miller JL, Vaughan AM, et al. Model for in vivo assessment of humoral protection against malaria sporozoite challenge by passive transfer of monoclonal antibodies and immune serum. Infect Immun. 2014 Feb;82(2):808-817. PubMed PMID: 24478094; PubMed Central PMCID: PMCPMC3911395.

43. Vaughan AM, Pinapati RS, Cheeseman IH, et al. Plasmodium falciparum genetic crosses in a humanized mouse model. Nat Methods. 2015 Jul;12(7):631-633. PubMed PMID: 26030447; PubMed Central PMCID: PMCPMC4547688.

44. Douglass AN, Kain HS, Abdullahi M, et al. Host-based prophylaxis successfully targets liver stage malaria parasites. Mol Ther. 2015 May;23(5):857-865. PubMed PMID: 25648263; PubMed Central PMCID: PMCPMC4427874.

45. Mikolajczak SA, Vaughan AM, Kangwanrangsan $N$, et al. Plasmodium vivax liver stage development and hypnozoite persistence in human liver-chimeric mice. Cell Host Microbe. 2015 Apr 8;17(4):526-535. PubMed PMID: 25800544; PubMed Central PMCID: PMCPMC5299596.

46. Wijayalath W, Majji S, Villasante EF, et al. Humanized HLA-DR4. RagKO.IL2RgammacKO.NOD (DRAG) mice sustain the complex vertebrate life cycle of Plasmodium falciparum malaria. Malar J. 2014 Sep 30;13:386. PubMed PMID: 25266106; PubMed Central PMCID: PMCPMC4197321.

47. Cosledan F, Fraisse L, Pellet $A$, et al. Selection of a trioxaquine as an antimalarial drug candidate. Proc Natl Acad Sci U S A. 2008 Nov 11;105(45):17579-17584. PubMed PMID: 18987321; PubMed Central PMCID: PMCPMC2579890.
48. O'Neill PM, Amewu RK, Charman SA, et al. A tetraoxane-based antimalarial drug candidate that overcomes PfK13-C580Y dependent artemisinin resistance. Nat Commun. 2017 May 24;8:15159. PubMed PMID: 28537265; PubMed Central PMCID: PMCPMC5458052.

49. Nilsen A, LaCrue AN, White KL, et al. Quinolone-3-diarylethers: a new class of antimalarial drug. Sci Transl Med. 2013 Mar 20;5 (177):177ra37. PubMed PMID: 23515079; PubMed Central PMCID: PMCPMC4227885.

50. Booker ML, Bastos CM, Kramer ML, et al. Novel inhibitors of Plasmodium falciparum dihydroorotate dehydrogenase with antimalarial activity in the mouse model. J Biol Chem. 2010 Oct 22;285 (43):33054-33064. PubMed PMID: 20702404; PubMed Central PMCID: PMCPMC2963363.

51. Skerlj RT, Bastos CM, Booker ML, et al. Optimization of potent inhibitors of P. falciparum dihydroorotate dehydrogenase for the treatment of malaria. ACS Med Chem Lett. 2011 Sep 8;2 (9):708-713. PubMed PMID: 24900364; PubMed Central PMCID: PMCPMC4018051.

52. Phillips MA, Lotharius J, Marsh K, et al. A long-duration dihydroorotate dehydrogenase inhibitor (DSM265) for prevention and treatment of malaria. Sci Transl Med. 2015 Jul 15;7(296):296ra111. PubMed PMID: 26180101; PubMed Central PMCID: PMCPMC4539048.

53. Kokkonda S, Deng X, White KL, et al. Tetrahydro-2-naphthyl and 2indanyl triazolopyrimidines targeting Plasmodium falciparum dihydroorotate dehydrogenase display potent and selective antimalarial activity. J Med Chem. 2016 Jun 09;59(11):5416-5431. PubMed PMID: 27127993; PubMed Central PMCID: PMCPMC4904246.

54. Phillips MA, White KL, Kokkonda S, et al. A triazolopyrimidine-based dihydroorotate dehydrogenase inhibitor with improved drug-like properties for treatment and prevention of malaria. ACS Infect Dis. 2016 Dec 09;2(12):945-957. PubMed PMID: 27641613; PubMed Central PMCID: PMCPMC5148661.

55. Norcross NR, Baragana B, Wilson C, et al. Trisubstituted pyrimidines as efficacious and fast-acting antimalarials. J Med Chem. $2016 \mathrm{Jul}$ 14;59(13):6101-6120. PubMed PMID: 27314305; PubMed Central PMCID: PMCPMC4947981.

56. Ibrahim N, Ibrahim H, Dormoi J, et al. Albumin-bound nanoparticles of practically water-insoluble antimalarial lead greatly enhance its efficacy. Int J Pharm. 2014 Apr 10;464(1-2):214-224. PubMed PMID: 24412521.

57. Jimenez-Diaz MB, Ebert $D$, Salinas $Y$, et al. (+)-SJ733, a clinical candidate for malaria that acts through ATP4 to induce rapid host-mediated clearance of Plasmodium. Proc Natl Acad Sci U S A. 2014 Dec 16;111(50):E5455-E5462. PubMed PMID: 25453091; PubMed Central PMCID: PMCPMC4273362.

58. McConville M, Fernandez J, Angulo-Barturen I, et al. Carbamoyl triazoles, known serine protease inhibitors, are a potent new class of antimalarials. J Med Chem. 2015 Aug 27;58(16):6448-6455. PubMed PMID: 26222445.

59. Baragana B, Hallyburton I, Lee $M C$, et al. A novel multiple-stage antimalarial agent that inhibits protein synthesis. Nature. 2015 Jun 18;522(7556):315-320. PubMed PMID: 26085270; PubMed Central PMCID: PMCPMC4700930.

60. Malmquist NA, Sundriyal S, Caron J, et al. Histone methyltransferase inhibitors are orally bioavailable, fast-acting molecules with activity against different species causing malaria in humans. Antimicrob Agents Chemother. 2015 Feb;59(2):950-959. PubMed PMID: 25421480; PubMed Central PMCID: PMCPMC4335868.

61. Le Manach $C$, Paquet $T$, Brunschwig $C$, et al. A novel pyrazolopyridine with in vivo activity in Plasmodium berghei- and Plasmodium falciparum-infected mouse models from structure-activity relationship studies around the core of recently identified antimalarial imidazopyridazines. J Med Chem. 2015 Nov 12;58(21):8713-8722. PubMed PMID: 26502160.

62. Le Manach C, Nchinda AT, Paquet $T$, et al. Identification of a potential antimalarial drug candidate from a series of 2-aminopyrazines by optimization of aqueous solubility and potency across the parasite life cycle. J Med Chem. 2016 Nov 10;59(21):9890-9905. PubMed PMID: 27748596. 
63. Paquet T, Le Manach C, Cabrera DG, et al. Antimalarial efficacy of MMV390048, an inhibitor of Plasmodium phosphatidylinositol 4kinase. Sci Transl Med. 2017 Apr 26;9(387). DOI:10.1126/scitranslmed.aad9735. PubMed PMID: 28446690.

64. Le Bihan A, De Kanter R, Angulo-Barturen I, et al. Characterization of novel antimalarial compound ACT-451840: preclinical assessment of activity and dose-efficacy modeling. PLoS Med. 2016 Oct;13(10):e1002138. PubMed PMID: 27701420; PubMed Central PMCID: PMCPMC5049785

65. Pegoraro S, Duffey M, Otto TD, et al. SC83288 is a clinical development candidate for the treatment of severe malaria. Nat Commun. 2017 Jan 31;8:14193. PubMed PMID: 28139658; PubMed Central PMCID: PMCPMC5290327.

66. Rueda L, Castellote I, Castro-Pichel J, et al. Cyclopropyl carboxamides: a new oral antimalarial series derived from the Tres Cantos Anti-Malarial Set (TCAMS). ACS Med Chem Lett. 2011 Nov 10;2 (11):840-844. PubMed PMID: 24900273; PubMed Central PMCID: PMCPMC4017974.

67. Sanz LM, Jimenez-Diaz MB, Crespo B, et al. Cyclopropyl carboxamides, a chemically novel class of antimalarial agents identified in a phenotypic screen. Antimicrob Agents Chemother. 2011 Dec;55 (12):5740-5745. PubMed PMID: 21968362; PubMed Central PMCID: PMCPMC3232824.

68. Hameed PS, Chinnapattu M, Shanbag G, et al. Aminoazabenzimidazoles, a novel class of orally active antimalarial agents. J Med Chem. 2014 Jul 10;57(13):5702-5713. PubMed PMID: 24914738.

69. Ramachandran S, Hameed PS, Srivastava A, et al. N-aryl-2-aminobenzimidazoles: novel, efficacious, antimalarial lead compounds. J Med Chem. 2014 Aug 14;57(15):6642-6652. PubMed PMID: 25007124.

70. Keurulainen L, Vahermo $M$, Puente-Felipe $M$, et al. A developabilityfocused optimization approach allows identification of in vivo fastacting antimalarials: N-[3-[(benzimidazol-2-yl)amino]propyl]amides. J Med Chem. 2015 Jun 11;58(11):4573-4580. PubMed PMID: 25906200.
71. Hameed PS, Solapure S, Patil V, et al. Triaminopyrimidine is a fastkilling and long-acting antimalarial clinical candidate. Nat Commun. 2015 Mar 31;6:6715. PubMed PMID: 25823686; PubMed Central PMCID: PMCPMC4389225.

72. Peters W. Drug resistance in Plasmodium berghei Vincke and Lips, 1948. I. Chloroquine resistance. Exp Parasitol. 1965 Aug;17(1):8089. PubMed PMID: 5843289.

73. Thompson PE, Bayles A, Olszewski B, et al. Quinine-resistant Plasmodium berghei in mice. Science. 1965 May 28;148(3674):12401241. PubMed PMID: 14280010.

74. Ibrahim N, Ibrahim $\mathrm{H}$, Sabater AM, et al. Artemisinin nanoformulation suitable for intravenous injection: preparation, characterization and antimalarial activities. Int J Pharm. 2015 Nov 30;495(2):671679. PubMed PMID: 26383839.

75. Rochford R, Ohrt C, Baresel PC, et al. Humanized mouse model of glucose 6-phosphate dehydrogenase deficiency for in vivo assessment of hemolytic toxicity. Proc Natl Acad Sci U S A. 2013 Oct 22;110(43):17486-17491. PubMed PMID: 24101478; PubMed Central PMCID: PMCPMC3808620.

76. Wickham KS, Baresel PC, Marcsisin SR, et al. Single-dose primaquine in a preclinical model of glucose-6-phosphate dehydrogenase deficiency: implications for use in malaria transmission-blocking programs. Antimicrob Agents Chemother. 2016 Oct;60(10):5906-5913. PubMed PMID: 27458212; PubMed Central PMCID: PMCPMC5038314.

77. Nanayakkara NP, Tekwani BL, Herath HM, et al. Scalable preparation and differential pharmacologic and toxicologic profiles of primaquine enantiomers. Antimicrob Agents Chemother. 2014 Aug;58 (8):4737-4744. PubMed PMID: 24913163; PubMed Central PMCID: PMCPMC4136050.

78. McCarthy JS, Marquart L, Sekuloski S, et al. Linking murine and human Plasmodium falciparum challenge models in a translational path for antimalarial drug development. Antimicrob Agents Chemother. 2016 Jun;60(6):3669-3675. PubMed PMID: 27044554; PubMed Central PMCID: PMCPMC4879391. 